EESTI NSV TEADUSTE AKADEEMIA TOIMETISED. 28. KOIDE FOUSIKA * MATEMAATIKA. 1979, NR. 4

ИЗВЕСТИЯ АКАДЕМИИ НАУК ЭСТОНСКОН ССР. ТОМ 28 ФИЗИКА * МАТЕМАТИКА. 1979, № 4

\title{
ХАРАКТЕРИСТИКИ ТИРИСТОРНО-ИНДУКТИВНОГО РЕГУЛЯТОРА ПЕРЕМЕННОГО НАПРЯЖЕНИЯ ПРИ ЕСТЕСТВЕННОЙ КОММУТАЦИИ
}

\author{
(Представлена И. Эпиком)
}

В настоящее время усилия исследователей направлены на создание регулируемых статических источников напряжения и тока с синусоидальной или близкой к ней формой выходного напряжения как наиболее рациональной для большинства потребителей электроэнергии переменного тока. В $\left[{ }^{1,2}\right]$ процессы работы одного из таких тиристорноиндуктивных регуляторов анализировались без учета величины индуктивности сглаживающего дросселя, которая принималась равной бесконечности. Однако, как показали результаты $\left[{ }^{3,4}\right]$, эта величина способна существенно изменять качество выходного напряжения. Поэтому в данной статье на примере тиристорно-индуктивного регулятора переменного напряжения, работающего при естественной коммутации тиристоров, - весьма перспективной в практическом отношении схемы из этого класса преобразователей - изучается влияние конечной величины индуктивности сглаживающего дросселя на характеристики схемы и конкретно определяется коэффициент гармоник выходного напряжения трехступенчатой формы в зависимости от угла управления и параметров схемы.

Принципиальная схема регулятора изображена на рис. 1. Для упрощения анализа все тиристоры приняты идеальными и пренебрежено активным сопротивлением и рассеянием дросселя. Нагрузка регулятора считается активной. Используются следующие обозначения и безразмерные параметры: $w_{1}, w_{2}, w_{3}$ - число витков отдельных секций дросселя; $L, L_{1}, L_{3}$ - индуктивности дросселя на соответствующих витках обмотки $\left(w_{1}+w_{2}+w_{3}\right),\left(w_{2}+w_{3}\right)$ и $w_{3} ; u_{1}(\theta)=\sin \theta-$ напряжение питания, где $\theta=\omega t$ и $U_{1 m}=1 ; i(\theta)=u(\theta)$ - ток и напряжение нагрузки $(R=1) ; \tau=\omega L / R-$ постоянная времени; $\alpha, \beta$ - углы отпирания и запирания тиристоров $T_{1}-T_{3}$.

Оптимальные параметры ступеней выходного напряжения, обеспечивающе подавление самых низких из высших гармоник, взяты из $\left[{ }^{5}\right]$ :

$$
B_{1}: B_{2}: B_{3}=1 /\left(w_{1}+w_{2}+w_{3}\right): 1 /\left(w_{2}+w_{3}\right): 1 / w_{3}=0,2610: 0,7135: 0,9745
$$

H

$$
\alpha_{2}-\alpha_{1}=\alpha_{3}-\alpha_{2}=\alpha_{5}-\alpha_{4}=\alpha_{6}-\alpha_{5}=\pi / 6, \alpha_{4}-\alpha_{3}=\pi / 3,
$$

где $B_{1}, B_{2}, B_{3}, \alpha_{1}=\alpha, \alpha_{2}, \ldots, \alpha_{6}-$ амплитуды и углы включения ступеней. 


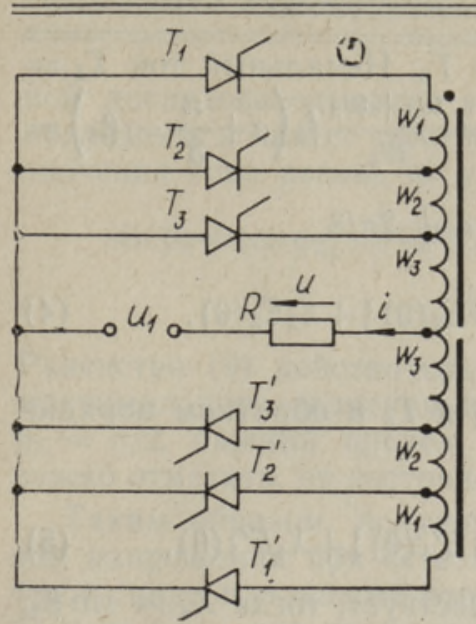

Рис. 1.

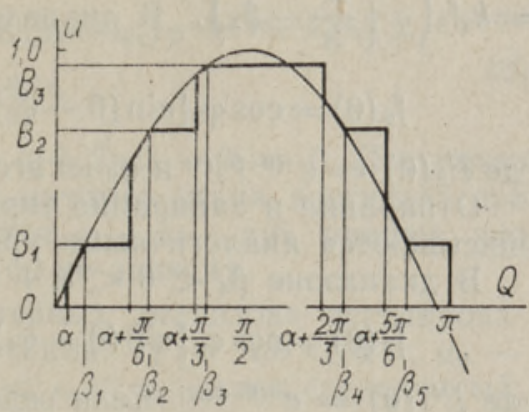

Рис. 2.

Анализ работы схемы проводится поэтапно методом припасовывания в предположений, что намагничивающая сила дросселя при коммутации неизменна. Ввиду симметричности рассматривается только положительный полупериод выходного напряжения. Уравнения записываются для случая существования всех этапов работы схемы, т. е. всех ступеней и синусоидальных отрезков в кривой выходного напряжения (рис. 2). С изменением угла управления $\alpha$ отдельные этапы в определенной последовательности могут исчезать.

П е р в ый эт а п - отперты тиристоры $T_{1}$ и $T^{\prime}{ }_{1}$. Имеет место при $\alpha<\theta<\beta_{1}$ и описывается уравнением

$$
i(\theta)=u(\theta)=f_{1}(\theta)=\sin \theta,
$$

которое справедливо также для третьего, пятого, седьмого и девятого этапов (этапов с синусоидальными отрезками) при отпертых состояниях тиристоров $T_{1}$ и $T_{2}$ или $T_{2}$ и $T_{3}$ в диапазонах $\alpha+\pi / 6<\theta<\beta_{2}$, $\alpha+\pi / 3<\theta<\beta_{3}, \alpha+2 \pi / 3<\theta<\beta_{4}$ и $\alpha+5 \pi / 6<\theta<\beta_{5}$ соответственно.

В торой э т п - отперт только тиристор $T_{1}$. Начальный ток $X_{1}=\sin \beta_{1}$. В диапазоне $\beta_{1}<\theta<\alpha+\pi / 6$

$$
f_{2}(\theta)=\cos \varphi\left[\sin (\theta-\varphi)+\sin \left(\varphi-\beta_{1}\right) E(\theta)\right]+X_{1} E(\theta),
$$

где $E(\theta)=\mathrm{e}^{-\left(\theta-\beta_{1}\right) / \tau}, \varphi=\operatorname{arctg} \tau . X_{1}$ определяется из условия отсутствия постоянной составляющей напряжения дросселя $\int_{\alpha}^{\alpha+\pi} u_{L}(\theta) d \theta=0$, где $u_{L}(\theta)=u_{1}(\theta)-u(\theta)-$ напряжение на его подключенных секциях. Ч е твертый $э$ та п - отперт только тиристор $T_{2}$. Начальный ток $X_{2}=\sin \beta_{2}$. Если третьего этапа нет, тогда $\quad X_{2}=\frac{w_{1}+w_{2}+w_{3}}{w_{2}+w_{3}} \times$ $\times f_{2}\left(\alpha+\frac{\pi}{6}-\beta_{1}\right)=k_{1} f_{2}\left(\alpha+\frac{\pi}{6}-\beta_{1}\right) . \quad$ В диапазоне $\beta_{2}<\theta<\alpha+\pi / 3$

$$
f_{4}(\theta)=\cos \varphi_{1}\left[\sin \left(\theta-\varphi_{1}\right)+\sin \left(\varphi_{1}-\beta_{2}\right) E_{1}(\theta)\right]+X_{2} E_{1}(\theta),
$$

где $E_{1}(\theta)=\mathrm{e}^{-\left(\theta-\beta_{2}\right) / \tau_{1}}$ и $\varphi_{1}=\operatorname{arctg}\left(\tau / k_{1}^{2}\right)$. 
Ш е с той э та п - отперт только тиристор $T_{3}$. Начальный ток $X_{3}=$ $=\sin \beta_{3}$. Если пятого этапа нет, тогда $\quad X_{3}=\frac{w_{2}+w_{3}}{w_{3}} f_{4}\left(\alpha+\frac{\pi}{3}-\beta_{2}\right)=$ $=k_{2} f_{4}\left(\alpha+\frac{\pi}{3}-\beta_{2}\right)$. В диапазоне $\beta_{3}<\theta<\alpha+2 \pi / 3$

$$
f_{6}(\theta)=\cos \varphi_{2}\left[\sin \left(\theta-\varphi_{2}\right)+\sin \left(\varphi_{2}-\beta_{3}\right) E_{2}(\theta)\right]+X_{3} E_{2}(\theta),
$$

где $E_{2}(\theta)=\mathrm{e}^{-\left(\theta-\beta_{3}\right) / \tau_{2}}$ и $\varphi_{2}=\operatorname{arctg}\left(\tau / k^{2}\right)$.

Отпирание и запирание тиристоров $T_{1}, T_{2}$ и $T_{3}$ в обратном порядке описываются аналогичными уравнениями.

В диапазоне $\beta_{4}<\theta<\alpha+5 \pi / 6$

$$
f_{8}(\theta)=\cos \varphi_{1}\left[\sin \left(\theta-\varphi_{1}\right)+\sin \left(\varphi_{1}-\beta_{4}\right) E^{\prime}{ }_{1}(\theta)\right]+X_{4} E^{\prime}{ }_{1}(\theta),
$$

где $E_{1}^{\prime}(\theta)=\mathrm{e}^{-\left(\theta-\beta_{4}\right) \tau_{4}}$. Если седьмой этап существует, тогда $X_{4}=\sin \beta_{4}$, если нет - тогда $X_{4}=\left(1 / k_{2}\right) f_{6}\left(\alpha+2 \pi / 3-\beta_{3}\right)$.

В диапазоне $\beta_{5}<\theta<\alpha+\pi$

$$
f_{10}(\theta)=\cos \varphi\left[\sin (\theta-\varphi)+\sin \left(\varphi-\beta_{5}\right) E^{\prime}(\theta)\right]+X_{5} E^{\prime}(\theta),
$$

где $E^{\prime}{ }_{1}(\theta)=\mathrm{e}^{-\left(\theta-\beta_{\vartheta}\right) / \tau_{1}}$. Если девятый этап существует, тогда $X_{5}=\sin \beta_{5}$, если нет - тогда $X_{5}=\left(1 / k_{1}\right) f_{8}\left(\alpha+5 \pi / 6-\beta_{4}\right)$.

При некотором предельном значении угла управления $\alpha_{\text {п }}$ этапы с синусоидальными отрезками исчезают, т. е. процессы работы регулятора при естественной и принудительной коммутации протекают совершенно одинаково.

Величина $\alpha_{\text {II }}$ в рассматриваемом случае определяется из условия

$$
\sin \left(\alpha_{\mathrm{n}}+2 \pi / 3\right)=X_{4}
$$

Используя результаты $\left[{ }^{4}\right]$ для определения $X_{4}$, можно найти зависимость $\alpha_{\text {II }}=f(\tau)$, показанную на рис. 3 , откуда видно, - что $\alpha_{\text {II }}$ изменяется мало при изменении $\tau$ в широких пределах. При $\tau=\infty$

$$
X_{4}=k_{1} X_{1}=2 k_{1} \cos \alpha_{\Pi} /\left[(\pi / 3)\left(1+k_{1}+k\right)\right]=\sin \left(\alpha_{\mathrm{n}}+2 \pi / 3\right),
$$

откуда

$$
\alpha_{\mathrm{I}}=\operatorname{arctg}\left[\sqrt{3}-12 k_{1} /\left[\pi\left(1+k_{1}+k\right)\right]\right]=18^{\circ} 27^{\prime} .
$$

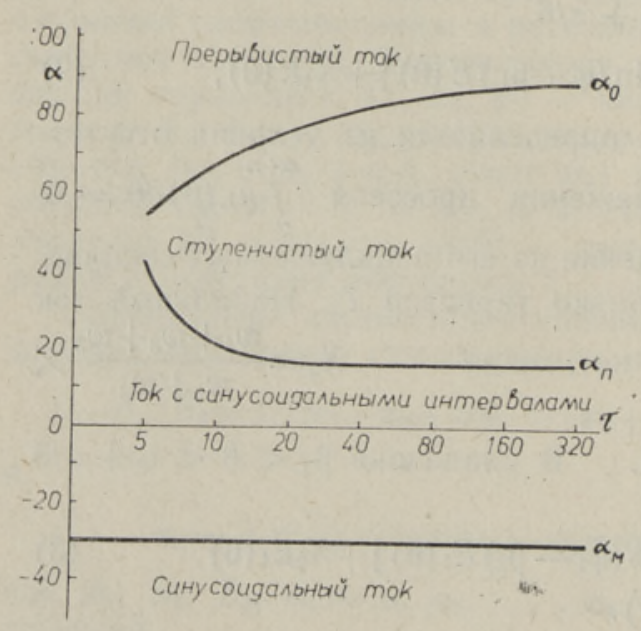

При $\alpha \geqslant \alpha_{0}$ в схеме возникает прерывистый ток, т. е. в некотором интервале времени этого режима все тиристоры заперты и ток нагрузки равен нулю. Соответствующая зависимость $\alpha_{0}=$ $=f(\tau)$ определена в $\left[{ }^{4}\right]$ и показана на рис. 3 .

С уменьшением угла управления при $\alpha \leqslant \alpha_{\text {n }}$ в схеме возникает ток с синусоидальными отрезками и продолжается до тех пор, пока в кривой выходного напряжения не исчезнут в определен-

Рис. 3 , 
ной последовательности все ступени. Принимая во внимание то, что последним исчезает шестой этап $\left[{ }^{2}\right]$ и при этом начальное и конечное значения тока равны, по уравнению (4) можем записать

$$
\begin{gathered}
\sin \beta_{3}=\cos \varphi_{2}\left[\sin \left(\alpha+2 \pi / 3-\varphi_{2}\right)-\sin \left(\beta_{3}-\varphi_{2}\right) \mathrm{e}^{-\left(\alpha+2 \pi / 3-\beta_{3}\right) / \tau_{2}}\right]+ \\
+\sin \beta_{3} \mathrm{e}^{-\left(\alpha+2 \pi / 3-\beta_{3}\right) / \tau_{2}} .
\end{gathered}
$$

Равенство (9) действительно только при $\alpha+2 \pi / 3-\beta_{3}=0$. Поскольку максимального значения начальный ток шестого этапа достигает при $\beta_{3}=\pi / 2$, нижний предел угла управления $\alpha_{\min }=-\pi / 6$, причем, что важно отметить, от постоянной времени $\tau$ он не зависит.

Таким образом, получены три характерных диапазона регулирования напряжения при естественной коммутации: 1) - $-\pi / 6<\alpha<\alpha_{\text {II }}$ ток с синусоидальными отрезками; 2) $\alpha_{\text {п }}<\alpha<\alpha_{0}-$ ток ступенчатый; 3) $\alpha>\alpha_{0}-$ ток прерывистый.

На основе приведенных выше уравнений можно найти коэффициенты искажения $k_{\text {и }}$ и гармоник $k_{\Gamma}$ по следующим формулам:

$$
k_{\mathrm{II}}=U_{(1)} / U=F_{1} / \sqrt{2} U
$$

и

$$
k_{\mathrm{r}}=\sqrt{1-k_{\mathrm{H}}^{2}} / k_{\mathrm{H}},
$$

где $U$ и $U_{(1)}$ - действующие значения выходного напряжения и его первой гармоники; $F_{1}=\sqrt{a_{1}^{2}+b_{1}^{2}} \quad$ - амплитуда первой гармоники выходного напряжения.

Амплитуды косинусоидального и синусоидального членов первой гармоники, а также действующее значение напряжения нагрузки выражаются формулами

$$
\begin{aligned}
& a_{1}=2 / \pi \int_{\alpha}^{\alpha+\pi} f(\theta) \cos \theta d \theta \\
& b_{1}=2 / \pi \int_{\alpha}^{\alpha+\pi} f(\theta) \sin \theta d \theta \\
& U=\sqrt{1 / \pi \int_{\alpha}^{\alpha+\pi} f^{2}(\theta) d \theta}
\end{aligned}
$$

где

$$
f(\theta)=\left.f_{1}(\theta)\right|_{\alpha} ^{\beta_{1}}+\left.f_{2}(\theta)\right|_{\beta_{1}} ^{\alpha+\pi / 6}+\left.f_{3}(\theta)\right|_{\alpha+\pi / 6} ^{\beta_{2}}+\left.f_{4}(\theta)\right|_{\beta_{2}} ^{\alpha+\pi / 3}+\ldots
$$

Соотношение (15) записано для случая существования всех этапов работы схемы (рис. 2). Если же некоторые из этапов исчезают в процессе регулирования, тогда соответствующие им подфункции $f_{k}(\theta)$ в (15) становятся равными нулю. Для диапазона $\alpha<\alpha_{\text {п }}$ характерны подфункции только с четными индексами, при этом коэффициент $k_{\Gamma}$ является наибольшим и, с точки зрения качества выходного напряжения, определяющим $\left(k_{\mathrm{r}}>0,151\right)\left[{ }^{4}\right]$. В диапазоне $\alpha<\alpha_{\text {п }}$ можно ограничиться вычислением $k_{\Gamma}$ при $\tau=\infty$. Как видно по крайним точкам pис. $4\left(\alpha=\alpha_{\text {п }}\right.$ и $\left.\alpha=-\pi / 6\right)$, величина $k_{\text {r }}$ не превышает найденной при $\tau=\infty$. 


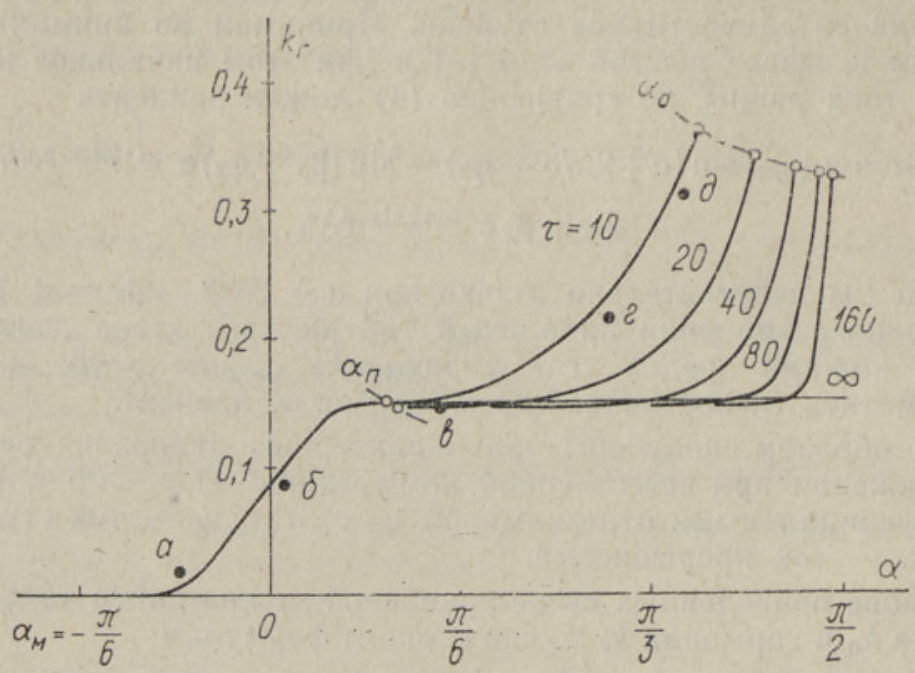

Рис. 4.

Согласно $\left[{ }^{2}\right]$, общее уравнение (15). действительно при изменении угла управления в пределах $-13^{\circ} 41^{\prime} \leqslant \alpha \leqslant 9^{\circ} 50^{\prime}$. В этом диапазоне коэффициенты ряда Фурье, а также действующее значение выходного напряжения при $\tau=\infty$ определяются по следующим формулам:

$$
\begin{gathered}
a_{1}=2\left\{\sin \beta_{1}[\sin (\alpha+\pi / 6)-\sin \alpha]+\sqrt{2} \sin \beta_{2} \cos (\alpha+\pi / 12)+\right. \\
\left.+\sin \beta_{3} \cos (\alpha+\pi / 6)-\sin ^{2} \beta_{1}-\sin ^{2} \beta_{2}-\left(\sin ^{2} \beta_{3}+\sin ^{2} \alpha+2\right) / 2\right\} / \pi, \\
b_{1}=2\left\{\left(\beta_{3}-5 \alpha\right) / 2+\left(\sin 2 \alpha-\sin 2 \beta_{3}\right) / 4+\sin \beta_{1}[\cos \alpha-\cos (\alpha+\pi / 6)]+\right. \\
\left.+\sqrt{2} \sin (\alpha+\pi / 12) \sin \beta_{2}+\sin \beta_{3}\left[\cos \beta_{3}+\sin (\alpha+\pi / 6)\right]\right\} / \pi, \\
U=\sqrt{\frac{\left[\left(\beta_{3}-5 \alpha\right) / 2+\left(\sin 2 \alpha-\sin 2 \beta_{3}\right) / 4+\right.}{(2 \alpha+\pi / 6) \times}}
\end{gathered}
$$

На рис. 5 показана зависимость $U=f(\alpha)$ при различных значениях $\tau$, откуда видно, что кратность регулирования выходного напряжения при заданном, максимально допустимом коэффициенте гармоник существенно зависит от постоянной времени силовой цепи.

В качестве примера на рис. $6, a-\partial$ изображены осциллограммы выходного напряжения при различных углах управления $\alpha$ для $\tau=10$, $k_{1}=2,33, k_{2}=1,5$ и $k=3,5$. Соответствующие им значения коэффициентов гармоник выходного напряжения нанесены на рис. 4. Некоторое расхождение расчетной и экспериментальной зависимостей $k_{\mathrm{r}}=f(\alpha)$ обусловлено различными значениями коэффициентов $k_{1}, k_{2} \cdot$ и $k$.

В заключение можно отметить следующее.

1. Предельный угол управления $\alpha_{\Pi}$, начиная с которого регулятор при естественной коммутации тиристоров работает точно так же, как и при принудительной, мало изменяется при изменении $\tau$ в широких пределах. 2. Нижний предел регулирования по углу управления $\alpha_{\min }$ (соответствует максимальному выходному напряжению) не зависит от постоянной времени $\tau$ и при трехступенчатой форме напряжения равняется $-\pi / 6$. 


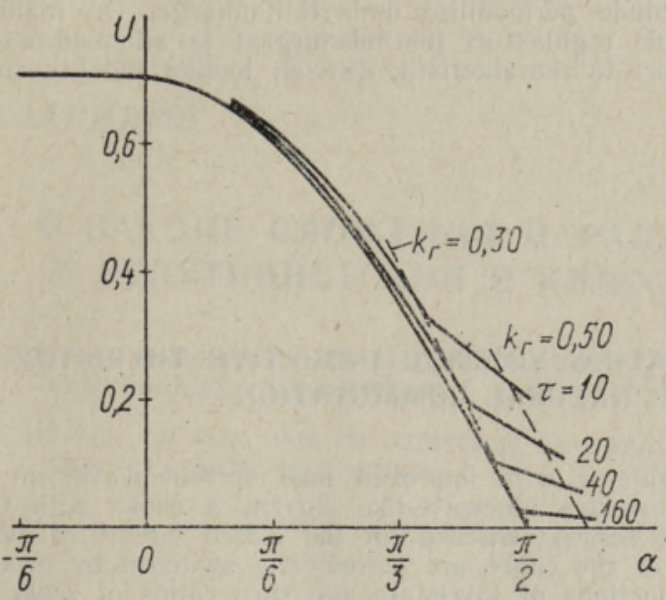

Рис. 5.

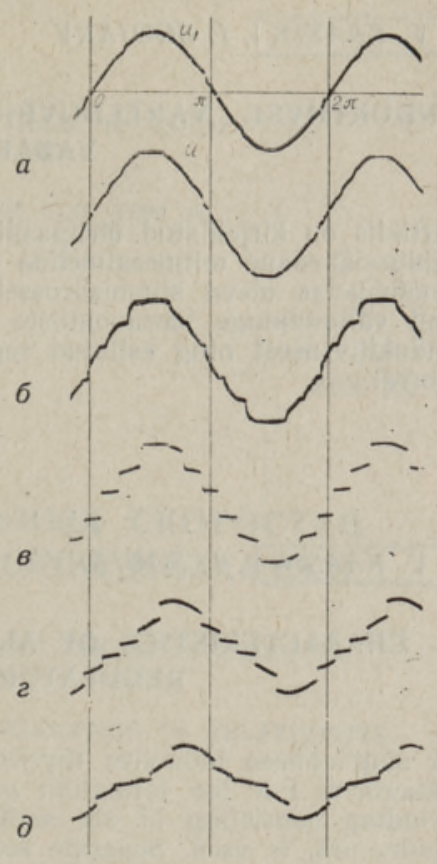

Рнс. 6.

3. При заданном, максимально допустимом коэффициенте гармоник $k_{\Gamma}$ диапазон регулирования выходного напряжения уменьшается с уменьшением постоянной времени $\tau$.

4. В диапазоне изменения угла управления $-\pi / 6 \leqslant \alpha \leqslant \alpha_{\text {п }}$ влияние $\tau$ на $k_{\Gamma}$ несущественно.

5. Важной особенностью данной схемы является то, что минимальные значения коэффициента гармоник соответствуют максимальным величинам выходного напряжения.

\section{Л ИТЕРАТ У РА}

1. О я в еэ р М., С ар в В., Изв. АН ЭССР, Физ. Матем., 26, № 3, 297-303 (1977).

2. О я в е эр М., С а р в В., Изв. АН ЭССР, Физ. Матем., 27, № 3, 342-348 (1978).

3. К а а з и к В., К а б р а л А., Изв. АН ЭССР, Физ. Матем., 27, № 3, 349-354 (1978).

4. К а азик В. Т., Сооя рв Ю. Э., В кн.: Теоретическая электротехника, Львов, Изд. Львовск. ун-та, 1979 (в печати).

5. Тонкаль С. С., В кн.: Проблемы технической электродинамики, вып. 24 , Киев, «Наук. думка», 1970, с. $37-42$.

Институт термофизики и электрофизики Академии наук Эстонской ССР
Поступила в редакцию

20/II 1979 
V. KAASIK I, J. SOOJARV

\section{INDUKTIIVSE VAHELDUVPINGE-TURISTORREGULAATORI KARAKTERISTIKUD VABAKOMMUTATSIOONI KORRAL}

Artiklis on kirjeldatud ühefaasilist induktiivset vahelduvpinge-türistorregulaatorit, milles siinuselähedane mitmeastmeline väljund- ja sisendvool formeeritakse regulaatori alaiisvooluahelas oleva silumisdrosseli keerdude perioodilise ümberlülitamisega. On määratud väljundpinge harmooniliste sõltuvus regulaatori juhtimisnurgast ja silumisdrosseli induktiivsusest ning esitatud regulaatori tüürkarakteristik drosseli lőpliku induktiivsuse korral.

\section{KAASIK| , J. SOOJARV}

\section{CHARACTERISTICS OF ALTERNATING VOLTAGE INDUCTIVE THYRISTOR REGULATOR WITH NATURAL COMMUTATION}

A single-phase inductive thyristor regulator with improved load current waveform is described. For the formation of a three-step sinewave-like current a choke with the winding consisting of six sections in series, switched on the closed circuit of even harmonics, is used. Separate sections of the choke are periodically switched by means of thyristor commutation. Existence functions of thyristors and turn ratios of separate sections are chosen such that in the case of choke inductance being equal to $L=\infty$ the lowest order of a significant harmonic in the load current is 11 th.

The main purpose of this paper is to determine dependence of control characteristics and load current distortions on choke inductance for a purely ohmic load under the conditions of natural commutation.

For desired current waveform the calculated control characteristics show a decrease of the variation range of output voltage with diminishing choke inductance. At the same time the firing angle corresponding to the maximum output voltage, is independent of choke inductance and for three-step current waveform is equal to $-\pi / 6$.

The calculated relationships between load current distortion ratio and firing angle at different values of choke inductance indicate that influence of choke inductance on current waveform is considerable only for small values of load current. Current distortion ratio increases with the decrease of both choke inductance and load current. 